\title{
EXUdATES DETECTION FROM Digital Fundus IMAGE OF DIABETIC RETINOPATHY
}

\author{
Namrata $^{1}$ and Shaveta Arora ${ }^{2}$ \\ ${ }^{1}$ Department of EECE, ITM University, Gurgaon, Haryana, India. \\ ${ }^{2}$ Department of EECE, ITM University, Gurgaon, Haryana, India.
}

\begin{abstract}
The aim of this paper is to detect exudates from the digital fundus images and provide information about Non Proliferative Diabetic Retinopathy. Diabetic retinopathy is very complicated disease that occurs when the retinal blood vessels changes. Exudates are the first sign of the diabetic retinopathy which cause blindness. So it is very important to find out these exudates in fundus image. In this paper we have proposed a method which is used for segmentation of optic disc and exudates. Morphological operations are used for detection of exudates. Before this operation we are applying Contrast Limited Adaptive Histogram Equalization technique. The results are compared with the standard database.
\end{abstract}

\section{KEYWORDS}

Diabetic Retinopathy, Fundus, Exudates, Dilation

\section{INTRODUCTION}

Diabetic retinopathy (DR) is one of the most common complications of diabetes and is one of the leading causes of blindness. In the retina, it can be characterized by blockage and damage of small blood vessels. A person who has diabetes for longer time then it is more likely that the person is suffering from this disease. If this disease not handle properly than it can interfere with patient's vision. In United States there are more than 4,000 people every year suffers due to Diabetic retinopathy. Diabetes is a chronic disease in which an individual stops producing and properly using of insulin. Diabetes can affect some patient organ such as kidney, nerve, heart and eye. Diabetes is a well-known disease and may cause abnormalities in the retina. This type of retinal abnormality is known as diabetic retinopathy. Diabetic retinopathy is microvascular abnormality which is caused by the diabetes. According to WHO (World Health organization) there will be 80 million people in India with diabetes by 2030 . As the number of diabetic patient increases so there is need of automated detection method for diabetic retinopathy.

In diabetes fundus imaging plays an important role for monitoring the retinal abnormalities such as Diabetic retinopathy. Useful clinical symptoms detected by fundoscopy are as follows:

\subsection{Micro aneurysms}

Micro aneurysms are a tiny aneurysm or swelling, in the wall of blood vessel. A person who has diabetes, Micro aneurysms are sometime found in the retina of the eye. These miniature aneurysms can rupture and leak blood (see in Figure 1.). In many research it can be indicated that these micro aneurysms can predict the progress of diabetic retinopathy, there is a condition in which blood vessels of the retina are damaged due to diabetes, which can cause blindness. Micro aneurysms are the first evident sign of non-proliferative diabetic retinopathy. So the first step is to recognize these retinal micro aneurysms. Retinal macro aneurysms are also an abnormality that can see in diabetic patient. It caused by any form of vascular disease or high blood pressure. It is more common in women than men. If these macro aneurysms forms in the macular area, so the 
patient has decreased central vision. If these macro aneurysms ruptures, so the patient has cloudy or little vision where the blood spreads.

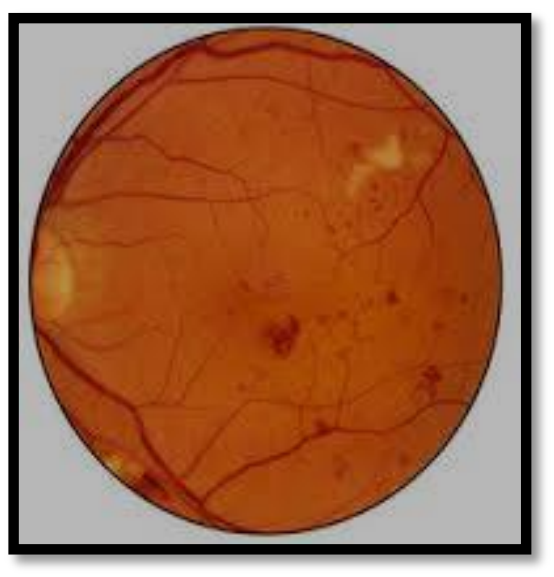

Figure 1.Microaneurysms [1]

\subsection{Dot and blot hemorrhage}

These hemorrhages arises slightly deeper, pre-venular, capillaries. In the middle layer of retina dot and blot like structures arises. It can cause hypertension, blockage of arterial vein. Blot hemorrhage are often large and darker. It occur very deeper layer of retina where the cells flow in vertical direction. It can also cause impairment of vision.

\subsection{Cotton wool spot}

In the funduscopic examination, there are finding some abnormality on the retina in the eye. It can be appear as yellow-white patch on the retina (see in Figure 2.). They are caused by damage to nerve fibers and when there is swelling in the surface layer of retina.

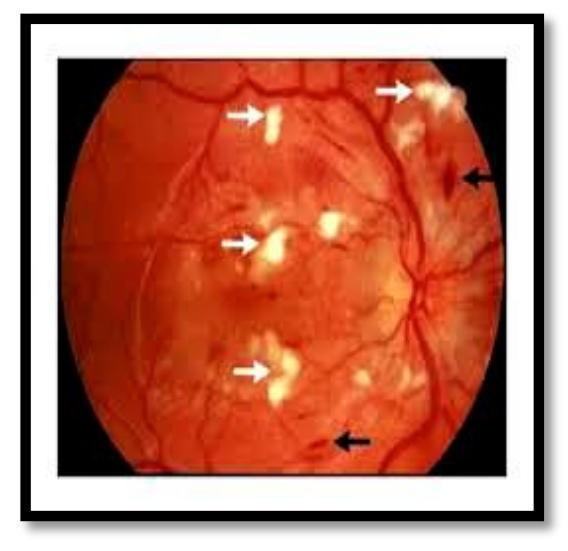

Figure 2.Cotton Wool Spot [2]

\subsection{Hard exudates}

Exudates are the lipid residues from serious leakage from damaged capillaries. There is a yellow fleck on the retina which is commonly known as exudates. In appearance they look shiny and waxy. It locates on the outer layer of retina. 


\section{Classification of Diabetic Retinopathy}

The diabetic retinopathy is classified in two levels and these levels are non proliferative diabetic retinopathy (NPDR) and proliferative diabetic retinopathy (PDR). The normal and abnormal retinal image is shown in Fig. 3(a). The earliest stage of diabetic retinopathy is Non proliferative diabetic retinopathy. Previously it is known as background retinopathy. It occurs when blood vessels of the retina is changed. These changes do not visible by the naked eye. In the early stage these symptoms do not noticeable but as it progress it also cause blindness. This type of diabetic retinopathy shows cotton wool spot, retinal hemorrhage and microvascular abnormalities. If there is development of macular edema so the NPDR person loses their vision. When there is swelling in the macula so it causes the fluid leakage from retina's blood vessel. In diabetes, the most common cause of vision loss is macular edema. If a person has diabetes with mild NPDR, in this the mild NPDR does not affect the vision of patient. If their vision is affected so, it is the result of macular edema.

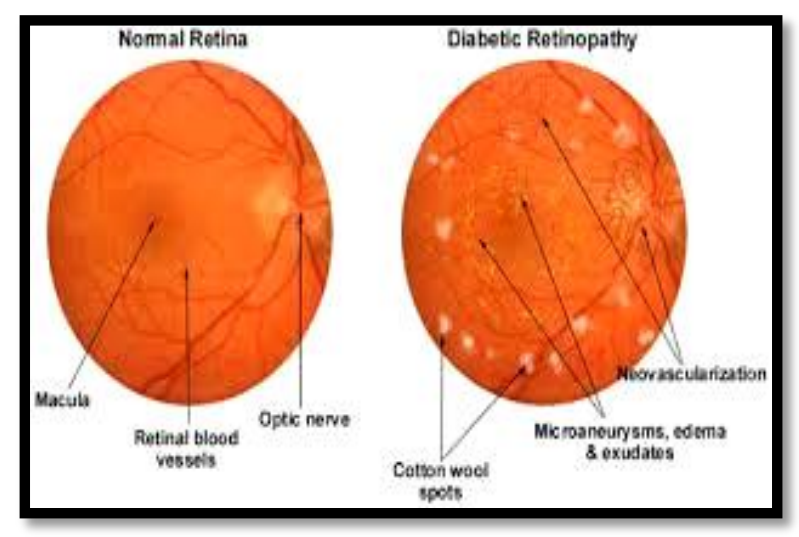

Figure 3(a).Normal and abnormal retinal image [3]

Non proliferative diabetic retinopathy also divided into three categories:

(a) Mild non proliferative retinopathy

(b) Moderate non proliferative retinopathy

(c) Severe non proliferative retinopathy

Proliferative diabetic retinopathy (PDR) in this type of retinopathy, some retinal blood vessels becomes block. Due to this blockage there is lack of blood in the retina and the new abnormal blood vessels growing in the retina (see in Fig. 3(b).). These newly grown blood vessels are fragile and may lead to bleed into the eye. Due to this abnormality it causes reduction in vision and sudden loss the vision of the eye. 


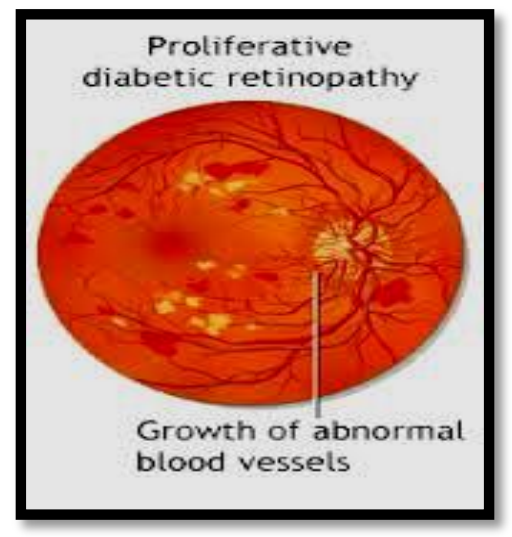

Figure 3(b).Proliferative diabetic retinopathy

\section{LITERATURE REVIEW}

Williamson et al. proposed a method in 2011 in which they used morphological operator for the detection of exudates with very low contrast image on diabetic retinopathy. In terms of sensitivity and specificity there results were evaluated [5]. Ramya et al. proposed a method in 2014 for automated detection of diabetic retinopathy. They used thresholding method for extraction of vessels of retina and use circular Hough transform method for detection of optic disc [6]. Faisal et al. proposed a method in 2014 using support vector machines (SVM). The results of this study showed $96.9 \%$ sensitivity and $100 \%$ specificity [7]. Murugan $\mathrm{R}$ et al. proposed method for extraction of microaneurysms with the help of mathematical morphology. The objective of this paper is to provide the overview of different methods of automated detection of microaneurysms using morphological operation. In this paper performance analysis is based on morphological operation is based on sensitivity and specificity [8]. Sinthanayothin et al. in 2001 proposed method for detection or extraction of features of diabetic retinopathy. They use recursive region growing algorithm with the help of moat operator. The result of this paper shows $88.5 \%$ sensitivity and $99.7 \%$ specificity [9]. Amrutkar et al. proposed a method in 2013 in which they used image segmentation and subtraction technique for exudates detection [10].

\section{Methodology}

We now describe the method which is proposed to detect the features of diabetic retinopathy.

\subsection{Pre-processing}

Pre-processing of retinal image is one of the most important steps. The main purpose of preprocessing is to improve the contrast of retinal image. Using this we can easily detect and localize the optic disc. There are many techniques have been employed for improving the contrast and color of the retinal image, but here we use Contrast Limited Adaptive Histogram Equalization (CLAHE). Contrast enhancement is the most important part of pre-processing. By this we can improve the contrast of the image. This histogram equalization technique enhances contrast of an image by transforming its intensity value. It operates on small region instead of 
the whole image [11]. If we have any invisible features in the image so, the contrast enhancement technique helps us to make these features more visible. In this we use CLAHE algorithm, this algorithm divide the image into different regions and then Histogram Equalization is applied to each and every regions.

First step is to acquire an original image. They are originally RGB in nature and then it is necessary to convert it into greyscale image. Now we move to the next step. In this step we are going to convert the original image into greyscale image. Our original image is RGB in nature but in this we extracted only green and greyscale image.

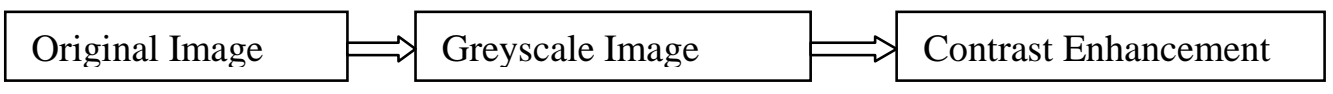

Figure 4.Flow chart for Pre-processing

\subsection{Removal of Optic Disc}

In retinal image, optic disc is the brightest characteristics of the fundus image. In appearance the color of optic disc is bright yellow in color fundus image. The contrast and color of the exudates and optic disc is very similar. So there is need to remove the optic disc from fundus image. The procedure for removal of optic disc is given below.

It is needed to take the pre-processed image for optic disc removal. After that we apply morphological operation on pre-processed image which adds the pixels to the boundaries objects in an image. It also grows the boundary of the mage. In this we use flat disk shaped structuring element. The dilation technique helps us to observe the region of optic disc more clearly. After that we have apply global thresholding on the dilated image. With the help of thresholding we can remove all the unwanted components.

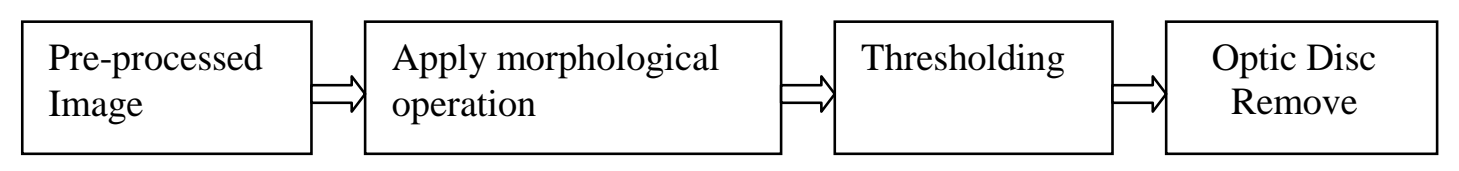

Figure 5.Flow chart for optic disc removal

\subsection{Exudates Detection}

After pre-processing and removing of optic disc, our objective is to detect the exudates. Median filter is widely used in image processing to eliminate noise. Here 2-D median filter is used to eliminate the noise while edges of an image are preserving. Median filter play an important role for proper detection of exudates. We apply median filtering on threshold image. After that we clearly detect the exudates.

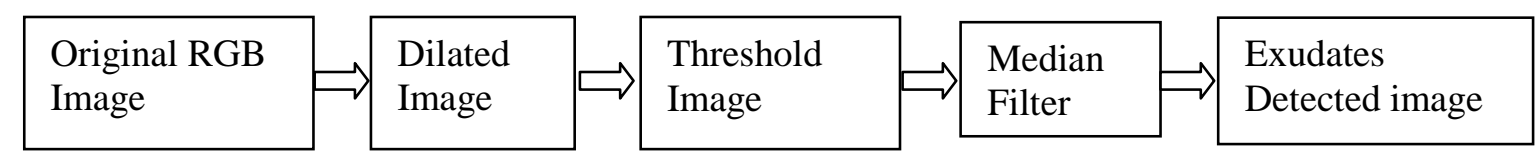

Figure 6.Flow for optic disc removal 


\section{Classification}

After detection of microaneurysms, we can classify that the fundus eye images are diseased or normal. This is depending upon the detected microaneurysms in the eye image. We classify the diabetic retinopathy into four stages and these stages are no DR, mild DR, moderate DR, and severe DR. Grade 0 is that stage when there is in no microaneurysms are detected so we can conclude that the person does not have diabetic retinopathy. Grade 1 is that stage where the number of microaneurysms lies between 1 and 5. This type of diabetic retinopathy is called mild diabetic retinopathy. Grade 2 is that stage when the number of microaneurysms increases and lies between 5 and 15 so $t$ is called moderate diabetic retinopathy. In grade 3 the number of microaneurysms is greater than 15 . This stage is known as severe diabetic retinopathy.

Table1. Grading of DR Stage

\begin{tabular}{|c|c|}
\hline Stage & Group \\
\hline Grade0(no DR) & MA $=0$ \\
\hline Grade1(mild DR) & $1<\mathrm{MA}<5$ \\
\hline Grade2(moderate DR) & $5<\mathrm{MA}<15$ \\
\hline Grade3(severe DR) & MA $>15$ \\
\hline
\end{tabular}

\section{RESULTS}

Here we use the dataset of retinal image which is publically available. We used more than 20 images for detection of exudates and the results of two normal and one abnormal fundus is shown here. Figure 7(a) and Figure 8(a) is the original RGB normal image and after applying pre-processing on the original image we get Fig. 7(b), 8(b), 7(c), 8(c), 7(d), 8(d) respectively. Figure 7(e) and Figure 8(e) is the final simulated image of normal eye image 1 and 2 respectively. There are no exudates detected so the patient does not suffer from diabetic retinopathy. Similarly, we apply the proposed algorithm on the Figure 9(a) and 10(a) abnormal retinal image 1 and 2 respectively so, we get the Figure 9(b), 10(b), 9(c), 10(c), 9(d), 10(d) respectively and finally we get Figure 9(e) and 10(e) which is the final image. In this image we detect the exudates so; the patient is suffering from diabetic retinopathy.

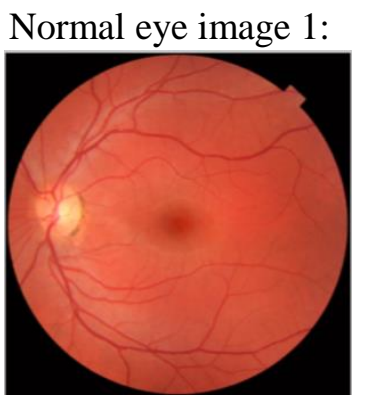

(a)

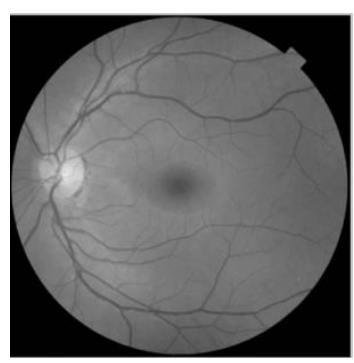

(b)

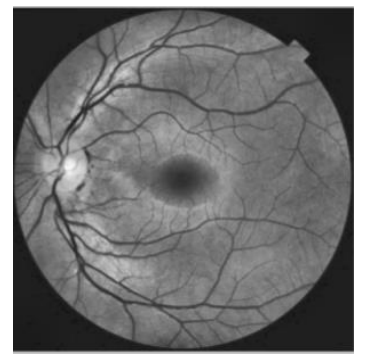

(c)

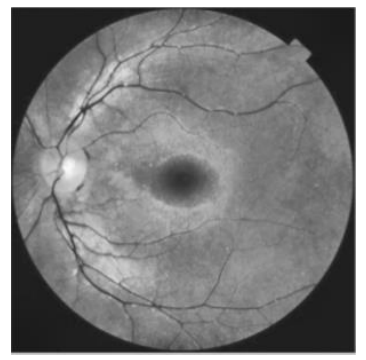

(d) 
International Journal of Advances in Biology (IJAB) Vol 2. No .4, November 2015

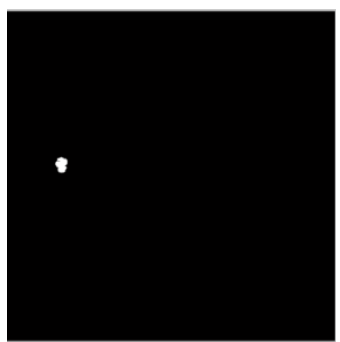

(e)

Figure 7(a).Original image, (b) Gray scale image, (c) Enhanced image, (d) Dilated image, (e) Final image.

Normal eye image 2:

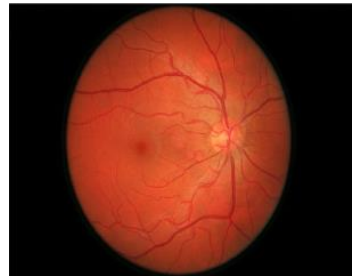

(a)

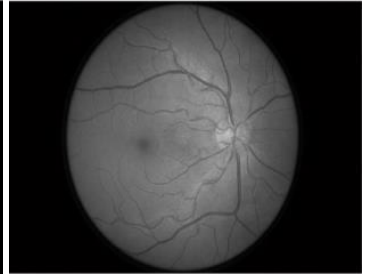

(b)

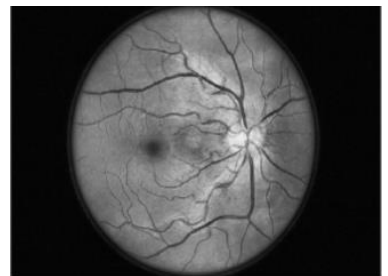

(c)

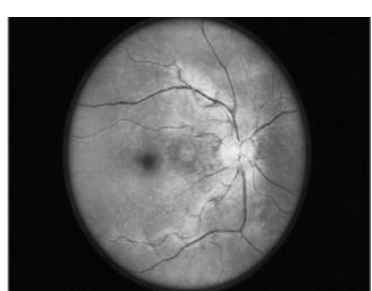

(d)

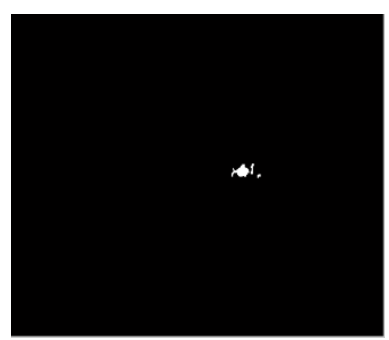

(e)

Figure 8 (a).Original image, (b) Gray scale image, (c) Enhanced image, (d) dilated image, (e) Final image Abnormal eye image1:

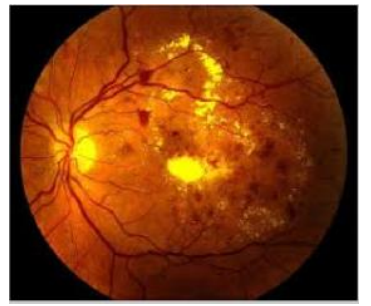

(a)

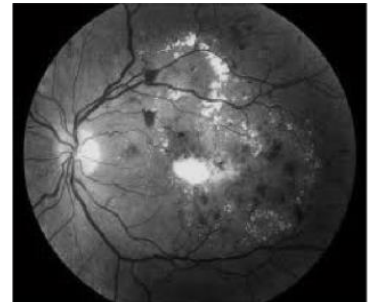

(b)

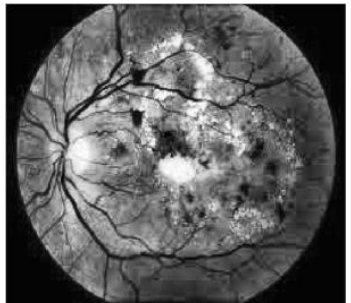

(c)

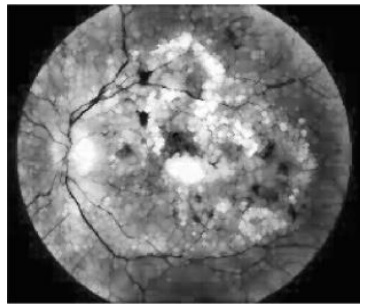

(d) 


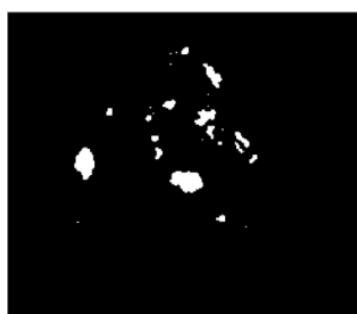

(e)

Figure 9 (a).Original image, (b) Greyscale image, (c) Enhanced image, (d) Dilated image, (e) Final image.

Abnormal eye image 2:

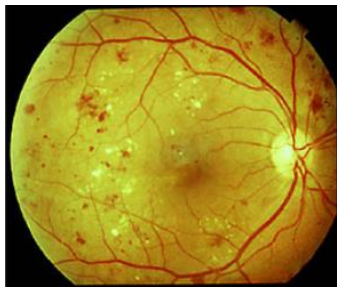

(a)

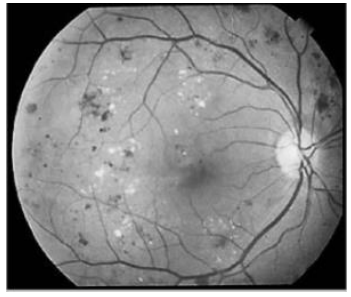

(b)

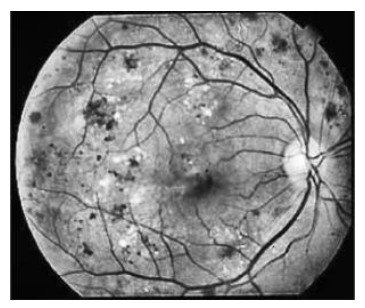

(c)

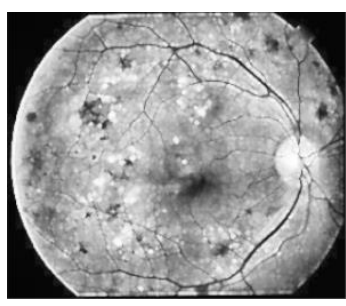

(d)

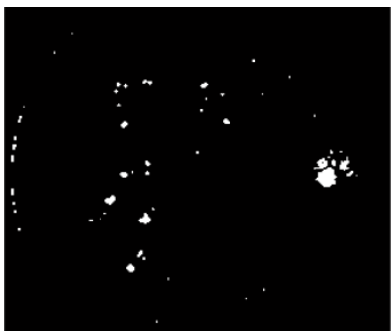

(e)

Figure 10 (a).Original image, (b) Greyscale image, (c) Enhanced image, (d) Dilated image, (e) Final image

\section{CONCLUSIONS}

The retinal color fundus image plays an important role in the detection of diabetic retinopathy. Diabetic retinopathy disease increases day by day. So it is very important to detect the early sign of diabetic retinopathy to reduce the chances of vision loss and blindness. In this we detect the exudates with the help of proposed algorithm. This method gives accurate and clear result. If it is require detecting the exudates then it must be prior to remove optic disc. Because the exudates and the optic disc have same color and contrast. This method is developed for detection of exudates which help the doctors in screening process of diabetic retinopathy.

\section{REFERENCES}

[1] Tanak Kampung Medical Info, Retinal fundus images [online] available:www.tapmed.blogspot.in/2012/10/diabetic-retinopathy.

[2] Digital fundus image [online] available: www.intranet.tdmu.edu.ua/data/kafedra/magistr.

[3] Retinal fundus image [online] available: http://www.rayur.com/diabetic-retinopathy. 
[4] Alireza Osareh, Bita Shadgar, and Richard Markham (4, July 2009) "A Computational-IntelligenceBased Approach for Detection of Exudates in Diabetic Retinopathy Images" IEEE Transactions on Information technology in Biomedicine, vol.13.

[5] Akara Sopharak, T. Williamson, S.Barman(2011) "Automatic microaneurysms detection from nondilated diabetic retinopathy retinal images" World congress of Engineering, WCE 2011, London,U.K.

[6] J.Ramya, S. Soundarya, E.Revathi(2014) "Detection of exudates in color fundus image"International journal of innovative research in Science, Engineering and Technology.

[7] M. Faisal, Djoko Wahono and M. Hariadi(2014) "Classification of diabetic retinopathy patients using support vector machines (SVM) based on digital retinal image" Journal of theoretical and applied information technology.

[8] Murugan R, Nasreen Fathima and Venketa Haritha T "Microneurysms detection methods in retinal images using mathematical morphology" International journal of advances in Engineering Science and Technology.

[9] C. Sinthanayothin, J. F. Boyce, H. L Cook, S.Lal(2001) "Automated detection of diabetic retinopathy on digital fundus image" Department of ophthalmology,St Thomas' Hospital, London, U.K

[10] Nikhil Amrutkar, Yogesh Bandgar, S.L Tade(2013) "Retinal blood vessel segmentation algorithm for diabetic retinopathy and abnormality detection using image subtraction" International journal of advance research in electrical, electronics and Instrumentation Engineering, vol.2.

[11] Hussain F.Jaafar, Asoke K. Nandi, Waleed Al-Nuaimy(2011) "Detection of exudates from fundus image using a region based segmentation techniques" European signal processing conference (EUSIPCO 2011). 